TITRE: SEIgNEURS MARCHANDS, ABOLITION ET MÉMOIRE : LE CAS DE LA SEIGNEURIE D'AUBERT-GALLION AutEUR: FrÉDÉRICK GOSSELIN, UNIVERSITÉ DE SHERBROOKE

PublicATION: LE RÉGIME SEIGNEURIAL AU QUÉBEC : FRAGMENTS D’HISTOIRE ET DE MÉMOIRE

PAGES: $105-124$

DIRECTION : BENOÎT GRENIER AVEC LA COLLABORATION D'AlAIN LABERGE ET DE STÉPHANIE LANTHIER ISBN: 978-2-7622-0361-5

URI: HTTP://HDL.HANDLE.NET/11143/16421

DOI: HTTPS://DOI.ORG/10.17118/11143/16421 


\title{
SEIGNEURS MARCHANDS, ABOLITION ET MÉMOIRE : LE CAS DE LA SEIGNEURIE D’AUBERT-GALLION
}

\author{
Frédérick Gosselin, candidat à la maîtrise en histoire, Université de \\ Sherbrooke.
}

Établi dès les débuts de la Nouvelle-France, le régime seigneurial, calqué sur les institutions françaises, est le principal moteur de développement durant les premières décennies de la colonie. Que ce soit pour mettre en valeur un nouveau territoire ou pour développer des activités commerciales, ce système foncier permet une croissance constante, mais inégale du territoire. Ainsi, les terres aux abords du fleuve Saint-Laurent sont les premières à être développées alors que les seigneuries dans l'est du Québec se caractérisent par un peuplement plus tardif'. Par ailleurs, le développement intensif à l'intérieur des terres, dont la région de la Beauce, ne s'effectuera qu'à la fin du régime français et surtout durant le régime britannique ${ }^{2}$. À ce titre, bien des seigneuries «beauceronnes» ont été concédées aux XVII et XVIII siècles, mais leur véritable développement ne se réalise qu'au XIXe siècle, et souvent sous l'égide de seigneurs anglophones. Par exemple, la seigneurie Aubert-Gallion fut concédée à François Aubert de La Chesnaye, fils de Charles Aubert, le 24 septembre $1736^{3}$. Selon Joseph Bouchette et plusieurs autres auteurs, cette terre est plutôt concédée directement à l'épouse de François Aubert, soit Marie-Thérèse de La Lande Gayon, d'où le fief tire son nom'. Toutefois, c'est sous la famille Pozer au XIXe siècle qu'Aubert-Gallion connait un véritable essor démographique et économique. Le poids mémoriel des Pozer est donc plus récent et plus imposant que celui des premières familles seigneuriales.

Sur le plan géographique, cette seigneurie fait face au fief voisin Aubin de l'Isle. Bouchette vante les mérites naturels d'Aubert-Gallion, exposant ainsi des «vastes pièces d'excellentes prairies $^{5}$ » de même que du «bois de construction de bonne qualité $e^{6}$. Concrètement,

1. Benoît Grenier, Seigneurs campagnards de la Nouvelle France: présence seigneuriale et sociabilité rurale dans la vallée du Saint-Laurent à l'époque préindustrielle, Rennes, Presses universitaire de Rennes, 2007, p. 17.

2. Serge Courville, Pierre C. Poulin et Barry Rodrigue, dir. Histoire de Beauce-Etchemin-Amiante, Québec, Institut québécois de recherche sur la culture, 2003, p. 118.

3. Ibid.

4. Joseph Bouchette, Description topographique de la province du Bas Canada : avec des remarques sur le Haut Canada et sur les relations des deux provinces avec les États-Unis de l'Amérique, W. Faden, Londres, 1815, p. 521.

5. Ibid.

6. Ibid. 
la concession seigneuriale se situe à proximité de la rivière Chaudière. Plus au sud, les Appalaches forment une frontière naturelle entre le territoire canadien et américain ${ }^{7}$. Cette chaine de montagnes dicte en quelque sorte l'établissement de la population et les secteurs d'activités. Ainsi, le sud est particulièrement boisé et montagneux, ce qui constitue un frein à l'exploitation agricole. C'est l'une des raisons pour lesquelles les habitants plus au nord de cette région seront essentiellement agriculteurs, activité favorisée par une idéologie dominante et par certaines plaines fertiles propices aux cultivateurs ${ }^{8}$.

Aubert-Gallion dispose également d'une caractéristique intéressante ; elle fut la possession de seigneurs anglophones émanant d'une classe marchande alors en pleine expansion après la Conquête. Soulignons d'entrée de jeu que le début du régime anglais permet une «marchandisation» de la propriété seigneuriale. Ainsi, nombre de marchands anglais ou écossais vont s'insérer dans le monde seigneurial en achetant des fiefs afin de faire fructifier leur investissement ou encore pour des questions de prestige. Sachant que deux des seigneurs d'Aubert-Gallion sous le régime britannique proviennent de la classe marchande, comment ce groupe social s'introduit-il dans les engrenages de la seigneurie d'Aubert-Gallion et comment celle-ci se distingue-t-elle des autres seigneuries «marchandes» tout au long de l'existence de la seigneurie et postérieurement à l'abolition de 1854 ? Nous soutenons ainsi que les seigneurs anglophones s'accaparent Aubert-Gallion pour des raisons différentes de telle sorte qu'il est difficile de façonner un modèle précis du seigneur marchand?. William Grant semble faire l'achat de la seigneurie pour une accumulation de titres alors que George Pozer met l'accent sur le développement de la seigneurie ${ }^{10}$.

De plus, la comparaison avec d'autres propriétaires marchands permet d'attester de l'unicité des intentions de chaque seigneur, et ce, même après la disparition du régime seigneurial $^{11}$. En examinant brièvement le parcours d'autres seigneurs marchands, il sera possible de conclure à une multitude de voies possibles, prouvant ainsi qu'il est impossible de définir un modèle unique du seigneur marchand. Par ce fait, l'histoire d'Aubert-Gallion ne se distingue pas des autres seigneuries achetées pas des marchands anglais, car chaque seigneur est porteur des motivations différentes ${ }^{12}$. L'abolition du régime seigneurial en 1854 pousse les Pozer à s'orienter vers le monde municipal en raison de l'influence de la famille au sein

7. Courville, Histoire de Beauce-Etchemin-Amiante, p. 27.

8. Ibid., p. 30.

9. Fernand Ouellet, «Propriété seigneuriale et groupes sociaux dans la vallée du Saint-Laurent (1663-1840)», Revue de I'Université d'Ottawa, vol. 47, n’ 1-2 (1977), p. 202.

10. David Roberts, « Grant, William (1744-1805) », Dictionnaire biographique du Canada » [en ligne], Consulté le 22 mars 2018, http://www.biographi.ca/fr/bio/grant_william_1744_1805_5F.html ; Louise Dechêne, «Pozer, George », Dictionnaire biographique du Canada » [en ligne], Consulté le 27 janvier 2018, http://www.biographi.ca/fr/bio/pozer_ george_7F.html.

11. Denis Vaugeois, Les premiers Juifs d'Amérique : l'extraordinaire histoire de la famille Hart, Québec, Septentrion, 2011, 378 p. ; André LaRose, «La seigneurie de Beauharnois 1729-1867, Les seigneurs, l'espace et l'argent», Thèse de doctorat (histoire), Ottawa, Université d'Ottawa, 1987, 685 p. ; Françoise Noel, «La gestion des seigneuries de Gabriel Christie dans la vallée du Richelieu (1760-1845) », Revue d'histoire de l'Amérique française, vol.40, n 4, 1987, p. 561-582.

12. Ouellet, « Propriété seigneuriale et groupes sociaux... », p. 202. 
de la communauté beauceronne. Il est possible d'y voir un aspect des persistances seigneuriales, car l'ancienne famille seigneuriale demeure influente dans les affaires municipales. La mémoire familiale continue cependant de persister après l'abrogation du régime. Aussi, la disparition de la notion marchande des premiers seigneurs et d'une mémoire favorable à l'endroit des Pozer est palpable au sein des descendants Pozer. Gordon et Charlotte Pozer insistent largement sur la bienveillance de leur famille ${ }^{13}$.

Nous diviserons ce texte en trois parties. La première explore l'intrusion des seigneurs anglophones dans le système seigneurial, suivi d'un court historique sur les principaux seigneurs d'Aubert-Gallion. La deuxième partie examine l'impact de la loi d'abolition du régime seigneurial en 1854 sur le fief des Pozer. Enfin, la mémoire des descendants Pozer sera mise à profit dans la dernière partie, en comparant leur mémoire avec les données historiques et en établissant des liens entre celles-ci.

\section{La mutation de la classe seigneuriale après la Conquête}

La victoire des Britanniques lors la guerre de Sept Ans change considérablement la composition ethnique et démographique de la province. La période d'occupation militaire (17591764) jette un voile d'incertitude sur l'avenir de la classe seigneuriale et du régime foncier en tant que tel. À cet égard, nombreux sont les propriétaires de fiefs qui vont se départir de leurs biens fonciers au profit de marchands anglais ${ }^{14}$. Ceux-ci vont graduellement percevoir l'acquisition de terres comme un revenu d'appoint parmi bien d'autres activités dont le commerce ou la spéculation financière. La question du prestige lié au titre de seigneur n'est également pas à négliger ${ }^{15}$. Pourtant, la composante bourgeoise du groupe seigneurial est déjà attestée sous le régime français comme le souligne Alain Laberge : « Cet intérêt tardif pour des fiefs à vocation agricole (car il ne faut pas oublier que des marchands ont possédé des seigneuries en dehors de la vallée laurentienne rurale comme en Gaspésie) révèle l'émergence de motivations économiques chez les marchands acquéreurs, intérêt qui ira en s'accentuant dans les décennies qui suivront les aveux et dénombrements ${ }^{16}{ }^{\prime}$. Laberge démontre également que la superficie des seigneuries selon la classe des seigneurs en 1725 atteste de l'existence de seigneurs marchands, bien que les nobles constituent la classe possédant la plus grande superficie de terre ${ }^{17}$.

13. Entretien 2017-04 avec Charlotte Pozer-Gamble, Saint-Georges de Beauce, $1^{\text {er }}$ aout 2017 ; Entretien $2017-06$ avec Gordon Pozer, Saint-Georges de Beauce, $1^{\text {er }}$ août 2017.

14. Benoît Grenier, Brève histoire du régime seigneurial, Montréal, Boréal, 2012, p. 157.

15. Ibid., p.156.

16. Alain Laberge, Jacques Mathieu et Lina Gouger, Portraits de campagnes: la formation du monde rural laurentien XVIII siècle, Québec, Presses de l'Université Laval, 2010, p. 83.

17. Ibid., p. 103. 
En fait, la classe seigneuriale à l'époque française est déjà plus hétérogène qu'homogène. D’abord, le titre de seigneur, bien que prestigieux, n'accorde théoriquement aucun avancement vers la noblesse. Pensons également à la présence de seigneurs issus de la classe paysanne ${ }^{18}$. Enfin, le dynamisme bourgeois est autant à l'honneur sous le régime français; la vente et la spéculation des terres étant chose courante ${ }^{19}$. Ainsi, la transition vers le régime anglais ressemble davantage à une mutation qu'à une rupture radicale. Ce sont les petits et grands marchands anglais qui vont devenir, en partie, les prochains seigneurs; l'évolution viendrait donc surtout de cette nouvelle composante ethnique.

Ce sont cependant les militaires anglais arrivés à la Conquête qui sont les premiers à s'insérer dans le monde seigneurial. À titre d'exemple, le gouverneur Murray achète sept fiefs durant son mandat ${ }^{20}$. Les administrateurs anglais seront poussés à tisser des liens avec les marchands britanniques qui vont rapidement accaparer de nouvelles terres ${ }^{21}$. Aussi, l'ancienne noblesse française qui demeure propriétaire de plusieurs seigneuries sera certes, bousculée par ces nouveaux venus, mais réussit pendant un certain temps à protéger ses intérêts fonciers. Le changement de régime n'apparait donc pas brutal en ce qui concerne le mode de tenure et les anciennes élites francophones continuent à jouer un rôle important dans la survie de ce système22.

Au début du XIX siècle, la survie du régime seigneurial devient de plus en plus précaire. Ainsi, le système de franc et commun socage, ou townships, établi par l'Acte constitutionnel de 1791 est l'une des premières mesures visant à restreindre le régime seigneurial ${ }^{23}$. L'augmentation de la population canadienne-française et la saturation des terres seigneuriales font en sorte que beaucoup de paysans s'orientent progressivement vers les terres en franc et commun socage et donc vers les townships. Cette situation inquiète les élites canadiennes-françaises qui y voient une attaque contre leur patrimoine identitaire ${ }^{24}$. Pour leur part, les marchands anglais ne favorisent pas nécessairement les townships, car le marché des seigneuries peut s'avérer fort lucratif. Les marchands anglais semblent s'inscrire graduellement dans la dynamique seigneuriale. À titre d'exemple, la spéculation des terres seigneuriales et l'octroi de prêts permettent à Aaron Hart d'acquérir un patrimoine foncier important dans la région de Trois-Rivières ${ }^{25}$. La question d'un revenu peu substantiel, mais constant, semble être à l'origine des motivations du marchand juif ${ }^{26}$. Comme nous le verrons, la seigneurie d'Aubert-Gallion ne fait donc pas exception à ce constat et s'insère direc-

18. Grenier, Brève histoire du régime seigneurial, p. 121-122.

19. Cameron Nish, «La bourgeoisie et le système seigneurial», L’Actualité économique, vol. 43, n’3, 1967, p. 509.

20. Ouellet, «Propriété seigneuriale et groupes sociaux... », p 198.

21. Roberts, « Grant, William (1744-1805) ».

22. Ouellet, «Propriété seigneuriale et groupes sociaux... », p. 208.

23. Grenier, Brève histoire du régime seigneurial, p.152.

24. Gérald Bernier et Daniel Salée, «Appropriation foncière et bourgeoisie marchande : éléments pour une analyse de l'économie marchande du Bas-Canada avant 1846 », Revue d'histoire de l'Amérique française, vol. 36, n’2, 1982, p. 168.

25. Vaugeois, Les premiers Juifs d'Amérique..., p.73.

26. Ibid., p. 131. 
tement dans cette logique commerciale accentuée par la conquête anglaise. William Grant et George Pozer, propriétaires successifs de la seigneurie, participent à cet intérêt pour la propriété foncière dans la nouvelle colonie, chacun disposant de caractéristiques qui leur sont propres.

\section{Le désintérêt de William Grant}

William Grant est né le 15 juin 1744 à Blairfindy en Écosse. Dès le début du régime anglais, Grant s'embarque vers la nouvelle colonie en vue d'établir des relations commerciales et y faire des investissements. Rapidement, il s’intéresse à la spéculation et à la traite des fourrures $^{27}$. II accumule ainsi une fortune qui lui permet de se procurer des terres et des propriétés partout dans la colonie. À cet effet, pensons à l'achat de la « seigneurie » de Saint-Roch ou encore à l'acquisition du château Ramezay pour 275000 livres $^{28}$. Incontestablement, Grant fait partie de cette classe de grands marchands britanniques qui profite de la paix et de l'ouverture du nouveau marché que représente l'ancienne colonie française. Ce groupe social va chercher au fil des années à promouvoir ses intérêts face aux décideurs politiques de la colonie. En fait, les nouvelles élites politiques et les marchands anglais et écossais entretiennent une certaine proximité les unes envers les autres, afin de défendre les intérêts de chacun ${ }^{29}$. C'est pourquoi Grant passe plusieurs années de sa vie dans la fonction publique. Son objectif premier: «[...] la mise en place d'un gouvernement représentatif et d'une réglementation du commerce impérial qui favoriserait les marchands de la coloni $\mathrm{e}^{30} »$.

Grant acquiert la seigneurie d'Aubert-Gallion en 1768 pour 100 livres. Cet achat s'insère dans une longue suite d'acquisition de propriétés. À cette époque, Aubert-Gallion est sous-développée, comme en témoigne sa faible valeur de revente. Il est difficile d'affirmer hors de tout doute les intentions de Grant quant à cet achat. Pensait-il en faire un investissement rentable à l'avenir? Le fait que Grant ne fasse arpenter cette seigneurie qu'en 1797 laisse croire que celle-ci n'occupait guère d'importance dans ses affaires. S'agissait-il dans ce cas d'une question de prestige? En 1768, il se marie avec Marie-Anne-Catherine Fleury Deschambault. Cette union lui permet d'acquérir la seigneurie de Longueuil, alors que cette terre porte le titre de baronnie ${ }^{31}$. Grant est donc le propriétaire de seigneuries bien plus développées et conséquemment plus prestigieuses; peut-être que l'accumulation de titres entre en ligne de compte, étalant ainsi ses titres ${ }^{32}$.

27. Roberts, « Grant, William (1744-1805)».

28. Ibid.

29. Grenier, Brève histoire du régime seigneurial, p. 147.

30. Ibid., p. 148.

31. Alex Tremblay-Lamarche, «La stabilisation et la créolisation de la présence seigneuriale britannique dans la vallée du Saint-Laurent, 1790-1815 », dans Benoît Grenier et Michel Morissette, dir., Nouveaux regards en histoire seigneuriale au Québec, Québec, Septentrion, 2016, p. 260.

32. Ibid., p. 261. 
Il est certain cependant que, pour Grant, l'achat des terres a une fonction économique : cela lui permet d'avoir un revenu continuel, alors que ses principales activités se situent dans les droits de pêche et la spéculation ${ }^{33}$. À cet égard, le parcours d'Alexander Ellice mérite d'être mentionné en raison du cheminement similaire entre les deux Britanniques. Marchand écossais arrivé à Montréal en 1776, il acquiert rapidement une fortune en spéculant sur la propriété foncière à la fois aux États-Unis et dans la nouvelle colonie anglaise ${ }^{34}$. L'appropriation terrienne pour ces deux seigneurs semble donc avoir une visée spéculative d'abord et de prestige par la suite. Par exemple, la seigneurie de Beauharnois est bien peu développée à l'époque et donc peu prestigieuse. C'est l'effort de peuplement qui rendra la seigneurie rentable ultérieurement ${ }^{35}$. De plus, l'augmentation du prix du blé favorise l'expansion de nouvelles terres en vue d'une exportation par les marchands. Le désir d'accroitre leurs avoirs par l'agriculture devient une motivation de plus en plus présente à la fin du XVIII siècle $^{36}$.

\section{Développement et peuplement sous les Pozer}

La mort de William Grant en 1805 provoque la mise aux enchères d'Aubert-Gallion en raison d'une faillite ${ }^{37}$. En 1807, Jean George Pozer se porte acheteur de la seigneurie d'Aubert-Gallion pour la somme de 550 livres. La faible augmentation de la valeur entre 1768 et 1805 prouve que le fief ne fut pas l'objet d'un développement intensif sous le seigneur Grant ${ }^{38}$. Le tout va changer considérablement avec l'arrivée de Jean George Pozer (1752-1848) comme propriétaire. C'est sous son impulsion et celle de son fils que la seigneurie va connaître un essor démographique et économique. Pourtant, Pozer provient d'un milieu relativement semblable à Grant : marchand d'origine allemande, Pozer s'installe d'abord à New York comme épicier avant de s'établir à Québec en 1785. Graduellement, ce dernier fait fortune dans l'import-export de bois ainsi que dans la spéculation et l'octroi de prêts ${ }^{39}$. Cela lui permet d'acquérir plusieurs propriétés immobilières dans la ville de Québec de même que la seigneurie de Saint-Étienne dans la région de la Beauce ${ }^{40}$.

En 1817, Pozer tente de développer Aubert-Gallion en invitant des colons allemands sur ses terres à ses frais afin d'y cultiver du chanvre ${ }^{41}$. La production de cette plante étant encouragée par les autorités, Pozer espère sans doute accroitre la production de sa nouvelle seigneu-

33. Roberts, Grant, « William (1744-1805)».

34. André LaRose, «La seigneurie de Beauharnois 1729-1867, Les seigneurs, l'espace et l'argent», Thèse de doctorat (histoire), Université d'Ottawa, 1987, p. 90.

35. Ibid., p. 92-93.

36. Tremblay-Lamarche, «La stabilisation et la créolisation... », p. 255.

37. Dechêne, «Pozer, George ».

38. Courville, Histoire de Beauce-Etchemin-Amiante, p. 186.

39. Ibid.

40. Dechêne, «Pozer, George ».

41. Ibid. 
rie $^{42}$. Cependant, l'implantation pour ces colons sera difficile, d'autant plus qu'un accident provoque un incendie et plusieurs de ses compatriotes décèdent ou quittent la seigneurie ${ }^{43}$. Ce fait n'est pas unique en soi, car d'autres seigneurs dont John Nairne ou Alexandre Fraser tentent d'établir dans leur seigneurie une population à leur image ${ }^{44}$. L'expérience de Pozer n'est pas singulière à l'échelle de la colonie et nous renseigne sur les motivations des seigneurs non francophones. Pour ces derniers, la nouvelle colonie anglaise semble symboliser une nouvelle opportunité pour leurs compatriotes.

Jean George Pozer résidera à quelques reprises dans sa seigneurie d'Aubert-Gallion à la différence de Grant. Ce point qui les distingue est peut-être l'élément qui permet de comprendre le développement un peu plus poussé par George Pozer et surtout par son fils William par la suite $^{45}$. Par exemple, le premier manoir seigneurial est construit sous William Pozer durant les années $1830^{46}$, alors que le moulin à farine est édifié par George vers $1818^{47}$. La clé pour expliquer une croissance soutenue dans la seigneurie semble être la présence du seigneur ou d'un agent vivant sur place, car les deux individus ont tout intérêt à faire fructifier leurs possessions. En effet, lorsque l'on compare la seigneurie d'Aubert-Gallion avec les seigneuries des Christie dans le Haut-Richelieu, la présence d'un acteur-clé sur place est essentielle: la famille Christie ne résidera jamais sur la seigneurie et transférera la gestion à un agent sur place ${ }^{48}$. Pourtant, les seigneuries de Bleury, Sabrevois, Noyan connaitront une expansion rapide, et ce, malgré l'absence du seigneur ${ }^{49}$. L'élément à retenir est la présence chez les Pozer et les Christie d'un membre (seigneur ou agent) dont l'objectif premier est le développement du fief. Cette notion est manquante dans le parcours de Grant et explique peut-être la faible croissance d'Aubert-Gallion sous son joug.

42. Ibid.

43. Claude Kaufholtz-Couture, «Une colonie allemande en Beauce autour d'un projet de culture du chanvre», Cap-aux Diamants, n’. 109, 2012, p. 16.

44. Tremblay-Lamarche, «La stabilisation et la créolisation... », p. 267.

45. Courville, Histoire de Beauce-Etchemin-Amiante, p. 187.

46. Ibid., p. 189-190.

47. Philippe Angers, Les seigneurs et premiers censitaires de St. Georges-Beauce et la famille Pozer, Beauceville, L’Éclaireur limitée, 1927, p. 15.

48. Françoise Noël, «La gestion des seigneuries de Gabriel Christie... », p. 565.

49. Ibid, p. 563. 


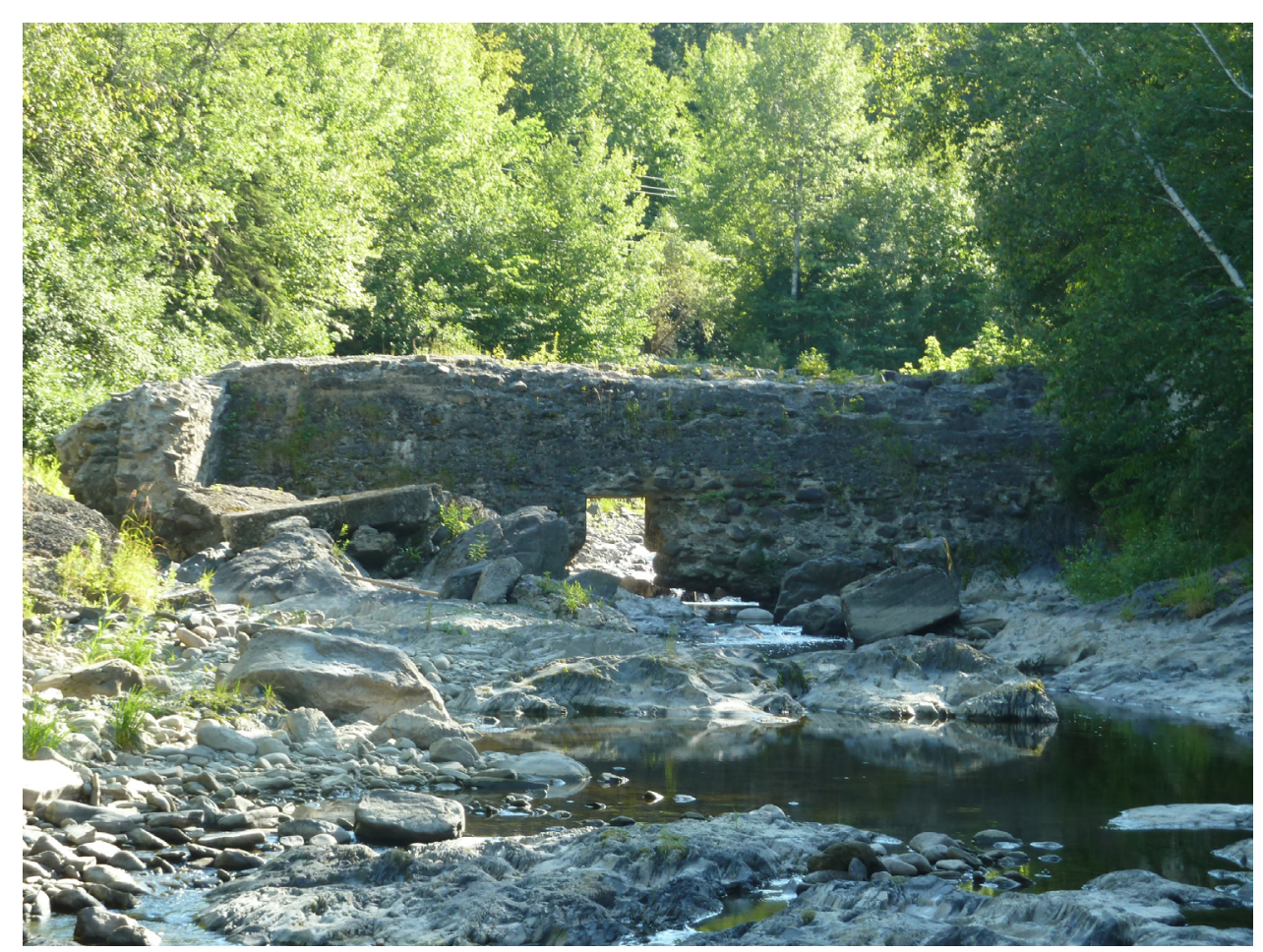

Photo 17 - Ruines du moulin seigneurial sur la rivière Pozer

Il ne semble donc pas y avoir de schémas explicatifs quant aux décisions des seigneurs marchands : ceux-ci vont agir différemment selon les besoins et les fluctuations du marché et de leurs seigneuries. L'activité marchande des seigneurs Pozer va graduellement s'estomper au fil du temps. Si le fils de George Pozer, Jacob, poursuit la même voie que son père en œuvrant dans l'import-export, c'est son dernier fils William qui deviendra le nouveau seigneur ${ }^{50}$. Celui-ci va demeurer dans la seigneurie de façon permanente. Pour autant, le commerce et le sens du développement ne semblent pas lui avoir échappé. En effet, il se préoccupe de la croissance de la seigneurie tant au niveau démographique que commerciale. Après l'échec du peuplement allemand, il tente d'attirer des colons canadiens-français ${ }^{51}$, encourage les voies de communication avec la route justinienne et plus tard la route Kennebec qui reliera les États-Unis à la ville de Québec ${ }^{52}$.

\section{Persistance légale de la tenure seigneuriale dans Aubert-Gallion}

Le fief des Pozer s'insère dans le contexte d'abolition et le cadastre abrégé de la seigneurie de même que les rapports du SNRSS nous permettent d'élaborer quelques pistes de réflexion sur la situation dans la seigneurie à cette époque charnière. La lecture du cadastre de

50. Angers, Les seigneurs et premiers censitaires de St. Georges-Beauce et la famille Pozer, p. 28.

51. Roger Bolduc, Saint-Georges : d'hier et aujourd'hui. Saint-Georges de Beauce, 1969, p. 19.

52. Honorius Provost, Chaudière-Kennebec: grand chemin séculaire, Québec, Éditions Garneau, 1974, p. 274. 
1857 réalisé par le commissaire Joseph-E. Turcotte éclaircit deux éléments majeurs de l'histoire de la seigneurie. D’abord, la composante multiethnique de la seigneurie est déjà effacée; les censitaires étant majoritairement d'ascendance française, outre les familles Munkell et Kahle, d'origine allemande ${ }^{53}$. Aussi, la dimension religieuse est visible dans ce cadastre puisqu'une concession est faite à l'évêque de Québec ${ }^{54}$. Ce fait est largement documenté dans les monographies locales où le seigneur Pozer offre des terres à l'église locale à même son domaine seigneurial. Ce dernier est délimité en 1854 à 18524 arpents $^{55}$. Il est sans doute possible que ce soit à partir de ce domaine que Charlotte Pozer évoque dans son témoignage un don de terre à l'hôpital local ${ }^{56}$.

Soulignons d'entrée de jeu qu'en 1940, la seigneurie est morcelée en quatre parties, comme le démontrent les quatre rapports pour les quatre réclamations demandées au SNRSS. Globalement, la valeur des cens et rentes était de 7497,50 \$ en 1857 alors que ce montant total sera de 6253,07 $\$$ en $1941^{57}$. Cette faible différence de valeur signifie que peu de gens ont racheté leurs rentes constituées et conséquemment que plusieurs des anciens censitaires s'acquittent encore de leurs rentes en 1941. Le tronçon de terre détenu par Peter William Langlois semble être la plus importante comme l'indique la compensation monétaire de $3539,53 \$$ à son égard ${ }^{58}$. Soulignons également que les héritiers de ce dernier possèdent des droits de propriété sur la seigneurie Saint-Étienne en Beauce ${ }^{59}$.

Les rapports du SNRRS sont peu loquaces en ce qui concerne la localisation des divisions au sein de la seigneurie. Seule une d'entre elles échappe aux mains des Pozer et devient propriété de Joseph Gagnon, marchand de Saint-Georges de Beauce. Ce même rapport d'octobre 1940 est le seul qui décrit l'emplacement du lot dans la seigneurie, soit la concession Saint-Guillaume, les 18 premiers numéros de la concession St-Jean Baptiste et une partie du premier rang ${ }^{60}$. Ce dernier acquiert sa partie de fief simplement par l'achat de ce terrain aux exécuteurs testamentaires de la veuve de William Milbourn Pozer, Mary Georgiana Pozer ${ }^{61}$. La seconde part datant de juillet 1941 est revendiquée par Peter William Langlois. Il conserve son titre de propriété de sa mère Barbara Johanna Pozer qui elle-même le détient de son frère Henry Christian Pozer ${ }^{62}$. Celui-ci est le fils de William Pozer, deuxième seigneur

53. Cadastres abrégés des seigneuries de Québec, Montréal, Trois-Rivières et de la Couronne, Québec, Derbishire et Desbarats, 1864, 7 volumes : seigneurie Aubert-Gallion.

54. Ibid.

55. Ibid.

56. Entretien 2017-04 avec Charlotte Pozer-Gamble, Saint-Georges de Beauce, $1^{\text {er }}$ aout 2017.

57. BAnQ-Québec, E39, S100, SS1, Fonds Syndicat national du rachat des rentes seigneuriales, Seigneurie Aubert Gallion.

58. Ibid.

59. Ibid.

60. Ibid.

61. Ibid.

62. BAnQ-Québec, E39, S100, SS2, Fonds Syndicat national du rachat des rentes seigneuriales, Seigneurie Aubert Gallion. 
d'Aubert-Gallion et d'Ann Milbourn. Il possède ainsi près de la moitié de l'ancienne seigneurie $^{63}$. La troisième requête date de novembre 1941 et résulte de la demande d'Ethel Jean Pozer qui sollicite 416,67 \$ pour ses droits seigneuriaux. Cette dernière est la fille de David Gordon Pozer qui acquiert l'héritage après la mort de son frère George Robert Pozer, luimême frère de William Milbourn Pozer ${ }^{64}$. La dernière réclamation (août 1941) provient de la succession de la veuve John Adolphus Pozer, M. Victoria Isabella Elliott. Le montant de cette requête est de 416,70 \$. La succession obtient donc ce titre de propriété directement de John Adolphus Pozer qui l'acquiert de son frère George Robert Pozer ${ }^{65}$.

À la lumière des renseignements obtenus lors du témoignage de Charlotte Gamble Pozer et de Gordon Pozer, ces derniers affirment que leur grand-père, George Alfred Pozer a agi à titre de seigneur ${ }^{66}$. Pourtant, celui-ci n'apparait nulle part dans la transmission des titres de propriété de la seigneurie selon les rapports du SNRRS. George Alfred Pozer est le fils de William Milbourn Pozer, qui est considéré comme le dernier seigneur d'Aubert-Gallion. Il est possible que George Alfred Pozer ait pu agir à titre de seigneur par intérim dans l'une des quatre divisions mentionnées plus haut. Au début du XXe siècle, la municipalité d'Aubert-Gallion est érigée et la fonction de maire semble importer davantage aux chroniqueurs locaux, comme en témoigne l'absence complète de George Alfred Pozer dans les ouvrages d'histoire locale ${ }^{67}$.

\section{Réorganisation des activités du seigneur et introduction au monde politique}

La sédentarisation de la famille Pozer semble avoir imposé un nouveau mode de vie. À partir de la troisième génération, l'activité marchande est délaissée, du moins pour les membres de la famille qui nous intéressent, au profit de la mairie locale. À titre d'exemple, le troisième seigneur d'Aubert-Gallion, William Milbourn Pozer sera maire en 1864-1865; son frère George Pozer le sera de 1872 à $1874^{68}$. Il est difficile d'affirmer hors de tout doute l'impact qu'a eu la loi sur la résiliation du régime seigneurial sur les activités des seigneurs Pozer. Est-ce en raison de cette loi que de nombreux membres de la famille se tournent vers les fonctions politiques plutôt que les activités purement marchandes? La municipalité d'Aubert-Gallion est érigée le 11 décembre 1856 par l'impulsion de l'abbé Antoine Campeau, en plein dans le contexte d'abolition du régime ${ }^{69}$. Il est possible que le rôle de seigneur perde simplement

63. Ibid.

64. Ibid.

65. BAnQ-Québec, E39, S100, SS1, Fonds Syndicat national du rachat des rentes seigneuriales, Seigneurie Aubert Gallion.

66. Entretien 2017-04 avec Charlotte Pozer-Gamble, Saint-Georges de Beauce, $1^{\text {er }}$ aout 2017.

67. Bolduc, Saint-Georges : d'hier et aujourd'hui. Saint-Georges de Beauce, p. 191.

68. Vézina, Histoire de Saint-Georges de Beauce, p. 113.

69. Bolduc, Saint-Georges : d'hier et aujourd'hui. Saint-Georges de Beauce, p. 46. 
de son importance et de son prestige au profit de la mairie ${ }^{70}$. On se doute que les seigneurs détenant des rentes constituées jouissent de ce revenu de façon secondaire, ce qui peut expliquer ce changement d'occupation ${ }^{71}$.

Une situation similaire est palpable chez les Fraser dans la nouvelle municipalité de Rivièredu-Loup $(1850)^{72}$. Cette famille possède la seigneurie grâce à son acquisition par Alexandre Fraser en 1802. Tout comme Aubert-Gallion, la seigneurie de Rivière-du-Loup se caractérise par son peuplement tardif, en raison d'un sol peu propice à l'agriculture. Dès l'abolition du régime seigneurial, les Fraser investissent ces nouveaux lieux de pouvoir que sont les municipalités. Quelques-uns des Fraser occupent la fonction de maire durant la deuxième moitié du XIXe siècle. À l'instar du seigneur qui bâtit des moulins ou des chapelles pour ses habitants, les Fraser construisent une église paroissiale en 1855 à même leur domaine ${ }^{73}$. Il est intéressant de voir que les Pozer agissent sensiblement de la même façon; les deux témoignages étudiés nous renseignent sur un don de terre de leur grand-père afin d'ériger une église et un hôpital ${ }^{74}$. Les détenteurs de pouvoir, du moins dans ces cas-ci, ne font que changer de titre. L'abolition du régime seigneurial est davantage une transition qu'une rupture et les anciennes élites deviennent les nouvelles élites. L'influence que possède la famille seigneuriale facilite la liaison vers le monde municipal. Cette dichotomie présente en quelque sorte une jonction entre tradition et modernité, sous la forme de seigneur/seigneurie par opposition à maire/municipalité.

L'analyse des rapports du SNRRS démontre à la fois l'état de la seigneurie en 1854 et l'impact qu'a pu avoir la loi sur l'abolition du régime seigneurial sur Aubert-Gallion. Le fief est divisé en quatre dont trois demeurent sous l'autorité de la famille Pozer. Toutefois, l'abolition des droits et des rentes seigneuriales force la famille seigneuriale à réorganiser ses activités afin de continuer à prospérer. L'influence qu'exercent les Pozer les pousse naturellement à occuper des fonctions de pouvoir, notamment au sein de la mairie de Saint George de Beauce. La prépondérance des Pozer dans les affaires de la nouvelle municipalité au-delà de 1854 suggère une persistance de la culture seigneuriale postérieure à son abolition. Cette notion est importante, car le témoignage des descendants des Pozer retient davantage l'implication municipale que le passé seigneurial ${ }^{75}$.

70. Michel Morissette, «L'argent et la propriété seigneuriale de 1854 à 1930 : qui sont les gagnants du processus d'abolition», dans Benoît Grenier et Michel Morissette, dir, Nouveaux regards..., p. 319.

71. Ibid., p. 318.

72. Benoît Grenier et Michel Morissette, «Propriétés et propriétaires seigneuriaux dans l'est du Québec entre 1854 et le milieu du XXe siècle : le cheminement comparé de l'île d'Anticosti et de Rivière-du-Loup », dans Harold Bérubé et Stéphane Savard, dir., Pouvoir et territoire au Québec depuis 1850, Québec, Septentrion, 2017, p. 51.

73. Ibid., p. 54.

74. Entretien 2017-06 avec Gordon Pozer, Saint-Georges de Beauce, $1^{\text {er }}$ août 2017.

75. Ibid. 


\title{
Les témoignages des descendants Pozer et la mémoire familiale
}

\author{
Source orale et distinction entre mémoire et histoire
}

Il est nécessaire de présenter d'abord l'apport que représentent les sources orales pour la compréhension du passé. Comme les sources orales proviennent du vécu d'individus particuliers, ce type d'information a longtemps été boudé par les historiens et les historiennes en raison de leur subjectivité ${ }^{76}$. L'oralité produit davantage une mémoire qu'une histoire scientifique à proprement parler. C'est pourquoi il convient de distinguer histoire et mémoire avant d'aborder de front la mémoire des descendants des Pozer.

Les sources orales sont essentielles, car elles peuvent nous renseigner sur certains aspects qui ne sont pas couverts par les études historiques. Soulignons la dichotomie qui existe entre histoire et mémoire. L'histoire est perçue comme étant la discipline scientifique dont l'objet d'étude est le passé. L'histoire est donc l'expérience rationnelle des humains envers leur passé ${ }^{77}$. Si l'on désigne souvent l'histoire comme étant froide et basée sur la raison, la mémoire est davantage fondée sur l'aspect chaud et sensible du passé. La mémoire fait partie de la construction identitaire de l'individu; c'est par la mémoire qu'un groupe se remémore le passé et qui devient par le fait même facteur de cohésion au sein de ce groupe. C'est lorsque l'histoire scientifique vient émettre certaines informations contredisant la mémoire qu'une friction se crée ${ }^{78}$. Cette mémoire peut prendre différentes formes, la plus connue étant sans doute la mémoire individuelle, issue des souvenirs d'une personne. Toutefois, il existe d'autres types de mémoires visibles dans notre environnement de tous les jours, dont la toponymie.

\section{Mémoire et persistance de la seigneurie Aubert-Gallion}

L’héritage de la famille Pozer a été marquant pour la municipalité de Saint-Georges de Beauce. Cette famille est perçue, à la fois par les ouvrages des chroniqueurs locaux que par la famille elle-même, comme étant la fondatrice de la ville. La toponymie de la ville de SaintGeorges est éloquente à ce sujet. Notons à titre d'exemple la présence d'une rivière, d'un parc, d'une école et même d'un cimetière portant le nom du fondateur de la municipalité. Le manoir seigneurial de William Milbourn Pozer datant de 1879 est désormais un restaurant

76. Vincent Duclert, «Archives orales et recherche contemporaine: Une histoire en cours », Sociétés \& Représentations, vol. 13, n' 1, 2002, p. 69.

77. Tzvetan Todorov, Les abus de la mémoire, Paris, Éditions Arléa, 2015, p. 24.

78. Ibid., p. 28. 
populaire dans la région et témoigne de l'existence marquée de cette famille seigneuriale ${ }^{79}$. Pensons également au nom même de la paroisse puis ville de Saint Georges de Beauce (1856), rappelant à la fois le nom de George Pozer et de Saint-Georges, martyr de l'Empire romain ${ }^{80}$.

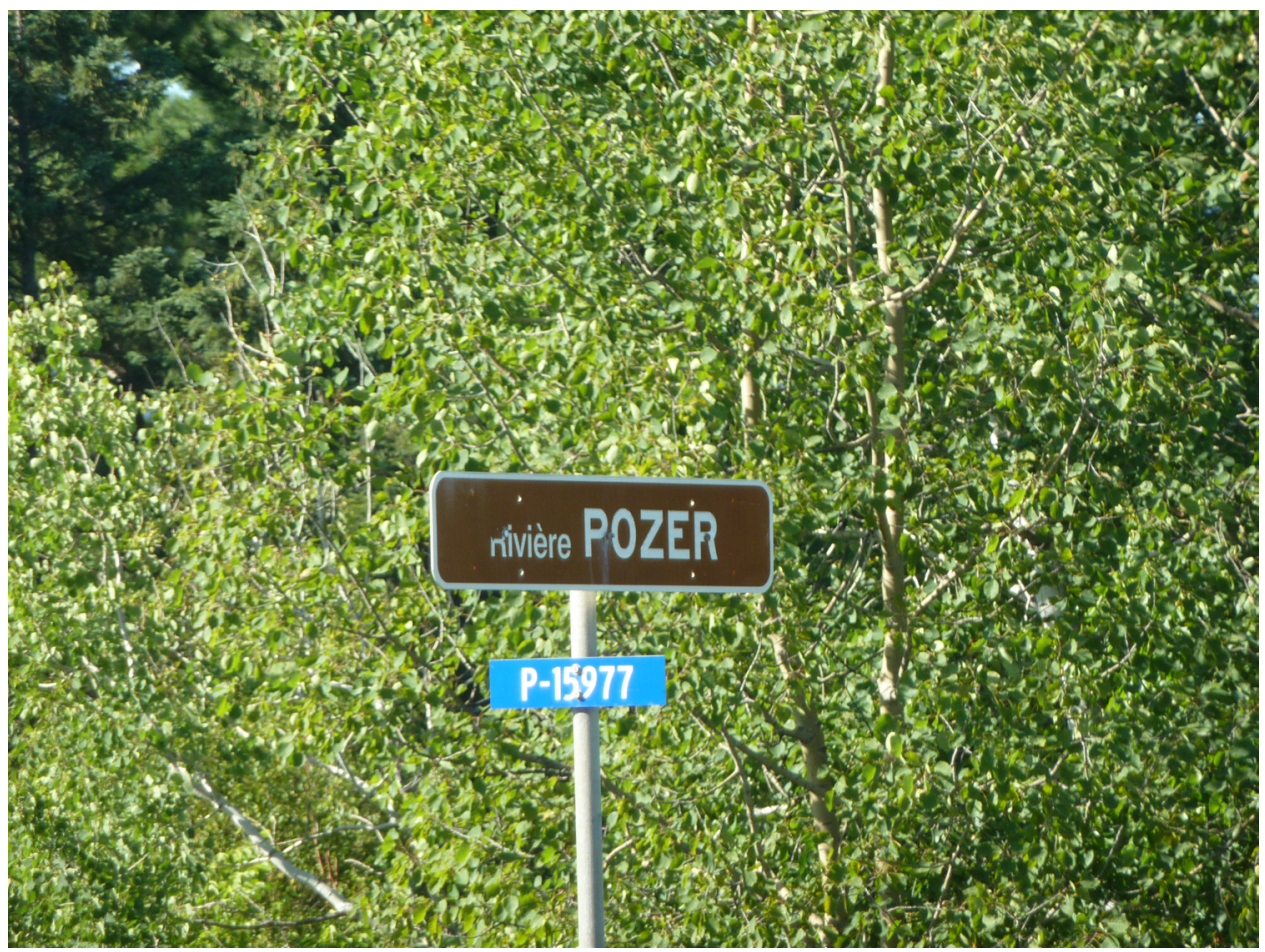

Photo 20 - La famille Pozer dans la toponymie de St-Georges

Cette section sera basée sur les entretiens de Charlotte Pozer Gamble et Gordon Pozer, réalisés par Benoît Grenier et Stéphanie Lanthier le $1^{\text {er }}$ août 2017 en Beauce. Descendants directs de la famille seigneuriale, ces deux témoins sont les enfants de Kenneth Pozer et Hariet Philbrick. Charlotte, née en 1932, et Gordon, né en 1938, sont les porteurs d'une mémoire familiale transmise au fil des générations. L'écoute de ces entrevues nous permet d'avancer que la mémoire familiale est plus importante que la mémoire seigneuriale. Par mémoire familiale, nous entendons la mémoire qui concerne uniquement la famille Pozer. En ce sens, les bonnes actions des Pozer, la dynamique familiale, l'interaction avec les habitants de Saint-Georges de Beauce sont mises en exergue, alors que la mémoire seigneuriale est mise de côté. En effet, rarement le nom d'Aubert-Gallion est-il mentionné, outre certains dons de terre. Aussi, l'activité marchande des premiers Pozer fait place à la conception municipale des Pozer. Selon l'entrevue des deux témoins, la mémoire familiale s'opère selon le cadre municipal, c'est-à-dire que Charlotte et Gordon Pozer insistent sur les actions de leur famille comme administrateurs de la ville. Ainsi, leur père qui dirige la mairie de Saint-Georges de

79. Répertoire du patrimoine culture du Québec, (2013), Manoir William-Milburn-Pozer (site web), Consulté le 30 mars 2017, http://www.patrimoine-culturel.gouv.qc.ca/rpcq/detail.do? methode=consulter\&id=92863\&type=bien\#. Wr6EDi7wbcs

80. Bolduc, Saint-Georges: d'hier et aujourd'hui. Saint-Georges de Beauce, p. 44. 
Beauce $^{81}$ est davantage reconnu comme étant un maire généreux plutôt qu'un descendant de la famille seigneuriale. Le même type de raisonnement s'applique à Charlotte et Gordon. En effet, ces derniers, bien que reconnus dans la municipalité, ne le sont pas en tant que descendant de la famille seigneuriale, mais plutôt comme fils et fille du maire de l'époque ${ }^{82}$. En somme, nous tenterons de démontrer que la notion marchande des premiers seigneurs s'estompe au profit des activités municipales des Pozer et de leur attitude remémorée comme bienveillante envers la population de Saint George de Beauce. C'est pourquoi la mémoire des descendants Pozer envers leurs ancêtres est davantage visible selon l'optique d'une mémoire familiale que d'une mémoire seigneuriale ${ }^{83}$.

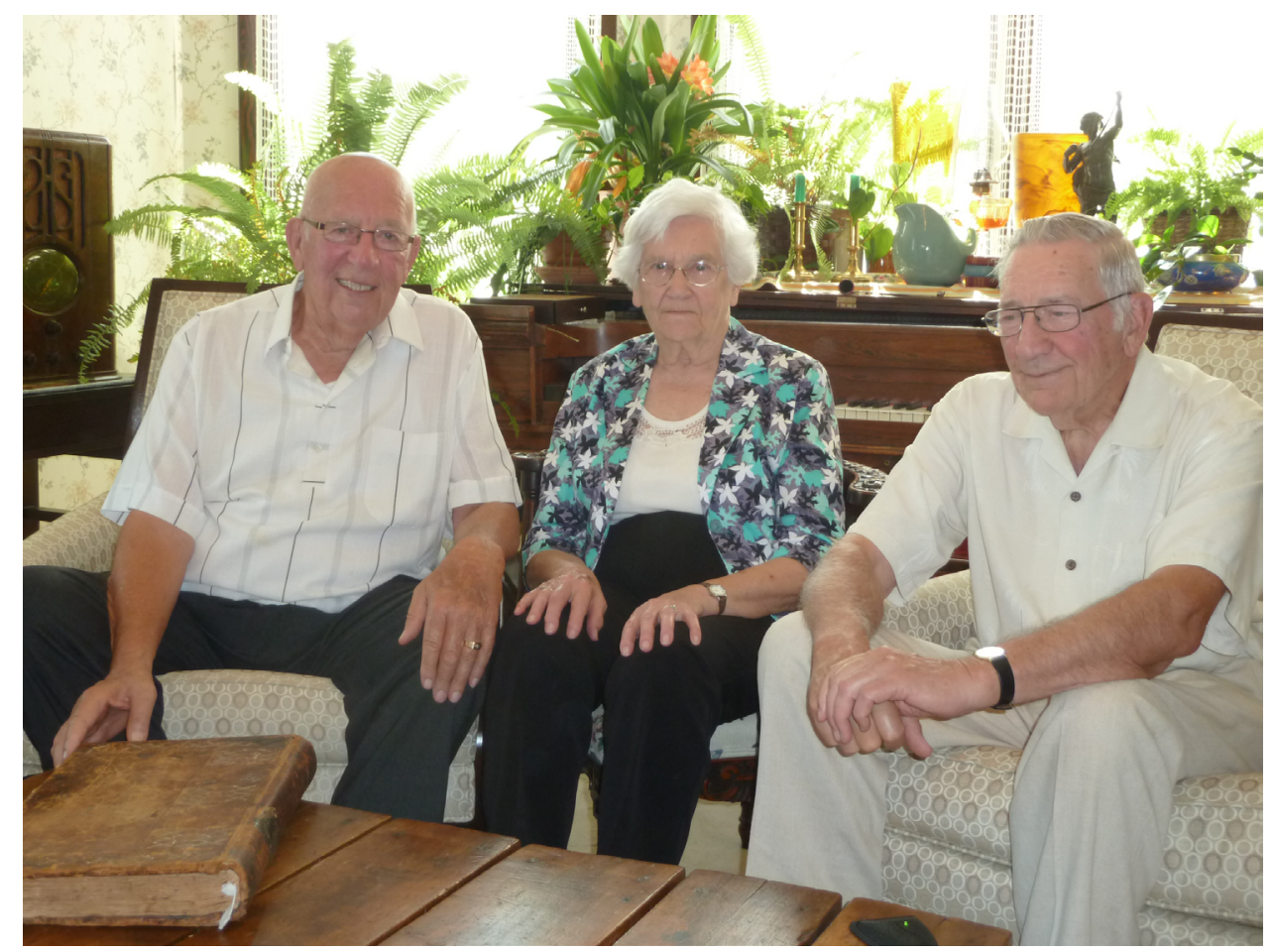

Photo 18 - Gordon, Charlotte et Dale Pozer, réunis à St-Georges pour livrer leurs témoignages, août 2017.

La mémoire des descendants des Pozer et des chroniqueurs locaux envers la famille Pozer est positive. Madame Charlotte Pozer le souligne : «j'étais fière de dire que mon nom c'était Charlotte Pozer $!^{84} »$. Cette fierté familiale est aussi fortement palpable chez Gordon qui affirme vouloir être enterré avec un contenant de terre provenant de la terre des Pozer d'Allemagne ${ }^{85}$. La volonté de transmettre le nom Pozer est importante pour les deux témoins. Gordon pousse la réflexion plus loin, en précisant sa déception que l'histoire locale ne soit pas plus enseignée: " c'est regrettable que l'histoire de Saint-Georges ne soit pas dans le

81. Entretien 2017-06 avec Gordon Pozer, Saint-Georges de Beauce, $1^{\text {er }}$ août 2017.

82. Entretien 2017-04 avec Charlotte Pozer-Gamble, Saint-Georges de Beauce, $1^{\mathrm{er}}$ aout 2017.

83. Ibid.

84. Ibid.

85. Entretien 2017-06 avec Gordon Pozer, Saint-Georges de Beauce, 1 ${ }^{\text {er }}$ août 2017. 
système d'éducation ${ }^{86} »$. De plus, Gordon associe une fierté importante au nom Pozer : « [...] c'est une fierté d'être un Pozer, et de marcher sur la 1re avenue et de dire bonjour tout le monde, et être traité pareil comme s'ils traitaient leurs proches. Même si on était anglophone, on était acceptés ${ }^{87}$ ». Pour Gordon, le nom Pozer semble être synonyme de camaraderie et de mansuétude au sein de la communauté beauceronne, soulignant le rôle fondateur de la famille et l'appréciation générale des concitoyens de Saint-Georges à propos des Pozer, d'où découle un sentiment fort de fierté.

Pour sa part, Charlotte déclare à plusieurs reprises durant son témoignage la bienveillance de son père et de sa famille auprès de leurs concitoyens. En ce sens, les gens respectaient les Pozer selon elle, d'autant plus que ses parents accueillaient charitablement ceux qui venaient s'acquitter de leurs dettes avec de la nourriture et des rafraichissements ${ }^{88}$. Cette vision positive transmise à la fois par les monographies locales et par la mémoire familiale se rapproche bien curieusement d'une représentation bienveillante du seigneur qui prend soin de ses censitaires, conception véhiculée par des auteurs tels que Philippe Aubert de Gaspé. Par exemple, Charlotte mentionne que les gens venaient demander des faveurs à son père, dont l'abstention de payer leur dû. Cette anecdote concorde quelque peu avec cette scène dans le roman Les Anciens Canadiens de Philippe Aubert de Gaspé où le censitaire Jean-Baptiste implore l'oncle Raoul de lui accorder son indulgence, car il ne peut payer ses rentes seigneuriales ${ }^{89}$. Seule nuance dans le cas des Pozer, la figure bienveillante du seigneur se transpose dans la fonction de maire et dans le paiement du lait dont ils étaient producteurs et distributeurs.

Autre fait intéressant, Philippe Angers écrit que Jean George Pozer, le premier des Pozer, lègue la majeure partie de ses possessions à sa fille Hannah et à son petit-fils George Alford au détriment de ses autres enfants. Cette succession trouble provoque une querelle familiale qui divisera les enfants de Jean George. Finalement, la question sera réglée par la reprise des titres de propriété par William Pozer ${ }^{90}$. Pourtant, Charlotte affirme lors de son témoignage que son père parlait peu des «choses de la famille». C'est peut-être en raison de cette dispute familiale et judiciaire qui s'est transmise au fil des générations.

Gordon et Charlotte soulignent également la donation faite par leur famille à la paroisse pour aider la communauté catholique, malgré le fait que la famille Pozer soit de confession protestante. Ainsi : « [...] on avait donné la moitié du terrain à l'Église et le couvent [...] donnait une classe anglaise. [...] II y avait d'autres petits enfants qui venaient pour apprendre l'anglais et moi j'étais bouche-trou pour la mathématique ${ }^{91}{ }^{\prime}$. Cette altérité entre anglo-

86. Ibid.

87. Ibid.

88. Ibid.

89. Philippe Aubert de Gaspé, Les Anciens Canadiens, Québec, Imprimerie Auguste Côté, 1877, p. 162-163.

90. Angers, Les seigneurs et premiers censitaires de St. Georges-Beauce et la famille Pozer, p. 24-25.

91. Entretien 2017-04 avec Charlotte Pozer-Gamble, Saint-Georges de Beauce, $1^{\mathrm{er}}$ aout 2017. 
phones et francophones et entre catholiques et protestants ne parait pas avoir été négative dans le cas d'Aubert-Gallion. À ce titre, Charlotte insiste sur sa participation dans la chorale catholique, son travail pour le curé, les dons de terres des Pozer pour cette même église et à l'hôpital afin d'illustrer la bonne cohabitation entre les deux ethnies et les deux cultes: « Quand on allait à l'hôpital, au début, avant que le gouvernement s'en mêle, on ne payait pas la chambre ${ }^{92}$ ». Gordon poursuit : « [...] c'est mon père qui a cédé le terrain pour l'hôpital ${ }^{93}$ ». Dans une optique plus négative, Charlotte raconte un épisode où un paroissien lui conteste le droit de s'asseoir sur la première rangée de bancs en raison de sa confession différente ${ }^{94}$. Outre cette anecdote, les relations entre seigneurs anglo-protestants et censitaires à majorité franco-catholique ne semblent pas avoir été une source de conflits majeurs ${ }^{95}$.

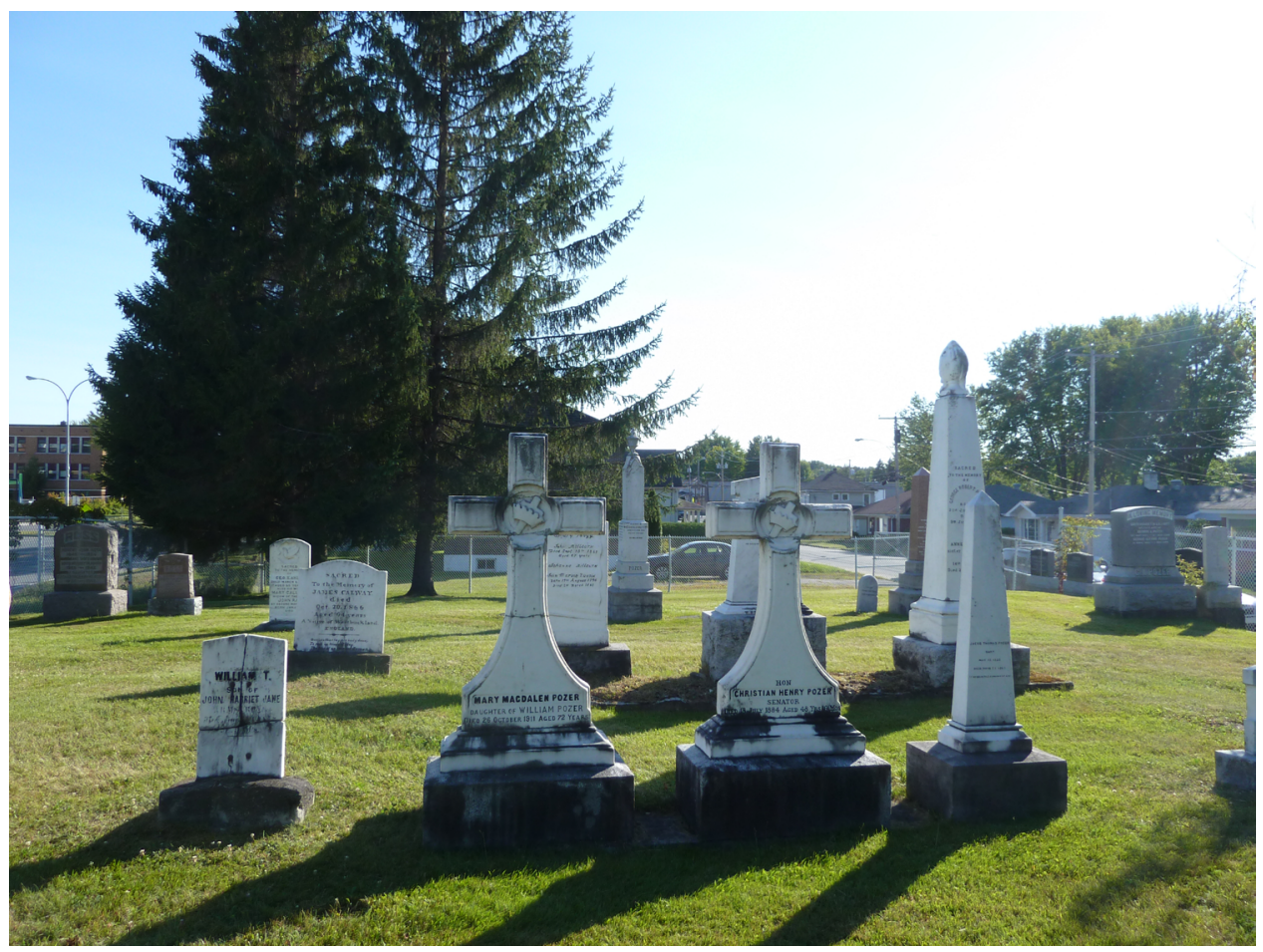

Photo 19 - Le cimetière « protestant » de la famille Pozer à St-Georges

Pourtant, mentionnons tout de même certaines tensions lors de la donation du seigneur George Pozer en 1824 pour l'édification d'une chapelle. Ainsi : "la construction est à peine commencée que les habitants adressent une requête à Mgr Plessis pour demander le transport de la chapelle sur le terrain de Joseph Rodrigue, voisin du domaine Pozer », et l'auteur de poursuivre: «Nos gens plaident leur cause avec chaleur, accusant l'abbé Primeau de "sympathies politiques à l'endroit des Pozer"96 ». Cette querelle quelque peu anecdotique vient relativiser certaines représentations mémorielles du passé, à la fois dans les monogra-

92. Ibid.

93. Entretien 2017-06 avec Gordon Pozer, Saint-Georges de Beauce, $1^{\text {er }}$ août 2017.

94. Entretien 2017-04 avec Charlotte Pozer-Gamble, Saint-Georges de Beauce, $1^{\text {er }}$ aout 2017.

95. Ibid.

96. Bolduc, Saint-Georges : d'hier et aujourd'hui. Saint-Georges de Beauce, p. 46. 
phies locales et dans la mémoire familiale qui idéaliseraient la cohabitation entre les deux confessions.

Le témoignage de Gordon expose une donnée intéressante; celui de la possession de la terre. Ce dernier affirme que son père achète une ferme après son emploi à la Banque Royale. S'agissait-il d'une portion de terre de l'ancienne seigneurie ${ }^{97}$ ? Nous avons mentionné les dons de terre effectués par Kenneth Pozer pour construire un hôpital, mais rien ne nous confirme qu'il s'agissait bien de l'ancien domaine seigneurial. Pourtant, les deux témoins affirment que leurs parents possédaient une portion de l'ancienne seigneurie. L'hypothèse selon laquelle Kenneth Pozer ait racheté une partie du domaine nous semble la plus plausible pour le moment, mais une visite dans les registres fonciers serait nécessaire.

L'activité commerciale des premiers seigneurs Pozer semble être oubliée par la mémoire familiale. C'est en partie le flair de George Pozer pour le négoce qui favorise initialement le développement de la seigneurie. Pourtant, ce moment crucial pour la croissance d'Aubert-Gallion ne semble pas être retenu par les descendants Pozer. Un peu à l'instar des chroniqueurs locaux, la mémoire récente retient mieux la politique locale comme étant le moteur de l'avancement de la région et au sein de la famille. À cet égard, Mme Pozer évoque constamment les réunions administratives qui se passaient dans le domicile familial, ou encore la charité de son père ou de sa famille envers ses concitoyens ${ }^{98}$.

\section{Conclusion}

En somme, la seigneurie d'Aubert-Gallion semble subir les effets de la Conquête; des marchands anglais s'intègrent dans les engrenages de ce fief en profitant d'un nouveau régime colonial. Bien que relativement nombreuse, cette classe sociale n'en est pas moins hétérogène. En effet, les marchands anglais et écossais acquièrent des seigneuries pour des raisons différentes, soit pour faire fructifier leurs investissements par le développement ou la spéculation ou encore pour des questions de prestige. Fernand Ouellet ajoute: «Entre le seigneur qui se contente de jouir de son statut, de jouer plus ou moins sur le niveau de la rente selon les circonstances, qu'elles soient économiques ou démographiques, et de la percevoir, et le seigneur capitaliste qui vise maximiser son profit, il existe toute une gamme d'attitudes ${ }^{99}$ ».

97. Entretien 2017-06 avec Gordon Pozer, Saint-Georges de Beauce, $1^{\text {er }}$ août 2017.

98. Entretien 2017-04 avec Charlotte Pozer-Gamble, Saint-Georges de Beauce, $1^{\text {er }}$ aout 2017.

99. Ouellet, «Propriétés seigneuriales et groupe sociaux... », p. 202. 
Ainsi, que ce soit la famille Ellice, Hart ou William Grant, la spéculation semble être l'une des raisons d'achat d'autant de propriétés terriennes. Pozer s'inscrit dans ce contexte, mais à l'instar des Hart, il installe sa famille dans sa seigneurie en vue de faire croitre son lopin de terre. Peut-être avait-il simplement l'intention de s'établir définitivement lui et sa famille dans sa seigneurie alors que les autres seigneurs évoqués précédemment comptaient davantage sur le retour d'investissement.

Nous avons évoqué dans cet essai la place du commerçant britannique comme nouvel acteur du système seigneurial d'après la Conquête, en essayant de démontrer les points semblables et distinctifs au sein de cette nouvelle classe sociale. Joseph Drapeau, riche commerçant qui fait fortune dans l'import-export, la propriété foncière et les créances fait office de contre-exemple en raison de ses origines canadienne-françaises. Rapidement, il s'intéresse aux terres seigneuriales comme moyen de faire fructifier ses avoirs. Le parcours de cet homme démontre que la marchandisation de la propriété seigneuriale n'est pas seulement l'œuvre des marchands anglais ou écossais ${ }^{100}$.

Toujours est-il que l'abolition du régime seigneurial coïncide avec un changement des activités des seigneurs. L'influence de la famille au sein de la paroisse maintenant devenue municipalité semble constituer une suite logique. Les anciens seigneurs Pozer seront en partie les prochains maires. La division quadripartite d'Aubert-Gallion à la fin du XIXe siècle démontre que les descendants des premiers Pozer demeurent en partie propriétaires de l'ancienne seigneurie. Le fait que les Pozer possèdent légalement la seigneurie pendant une cinquantaine d'années seulement prévient l'éclatement des possessions et les subdivisions du fief.

Cette conception municipale des activités des Pozer est probablement l'élément qui surgit le plus des témoignages de Charlotte et Gordon Pozer. Ce fait est sans doute explicable en raison de la proximité de cette mémoire; leur père ayant lui-même été maire de SaintGeorges-Ouest. Au contraire, la dimension marchande n'est nulle part mentionnée malgré l'importance que cette activité occupe pendant les deux premières générations. La mémoire familiale demeure favorable à son passé; pensons seulement à la bonne entente entre les seigneurs anglo-protestants et les censitaires majoritairement franco-catholiques ou encore à la sympathie des Pozer à l'endroit des autres citoyens. Il est d'autant plus intéressant que la mémoire familiale largement véhiculée par les deux témoins soit semblable à la mémoire provenant des monographies locales. De ce fait, les deux vantent les mérites de la famille fondatrice de Saint-Georges de Beauce. Cette mémoire demeure tout de même visible dans la toponymie locale.

100. Céline Cyr et Pierre Dufour, «Drapeau, Joseph », Dictionnaire biographique du Canada » [en ligne], Consulté le 14 janv. 2019, http://www.biographi.ca/fr/bio/drapeau_joseph_5F.html Le mémoire que prépare Raphaël BergeronGauthier à propos de Joseph Drapeau devrait permettre de mieux comprendre les conditions de la réussite de ce seigneur. 
Retenons en définitive qu'il n'y a pas de modèle du seigneur marchand après la Conquête puisque chaque propriétaire agit selon ses désirs et ses intentions. Les comparaisons de différents seigneurs permettent d'attester de ce fait. Cette notion marchande disparait au fil des années au profit de la fonction de maire, du moins dans le cas des Pozer. L'héritage des seigneurs d'Aubert-Gallion dans la municipalité de Saint-Georges de Beauce est donc majeur, mais en même temps complexe. La persistance seigneuriale a longtemps été palpable dans ce coin de pays à la fois par la voie légale et dans les mœurs. Aujourd'hui, outre la mémoire familiale des descendants des seigneurs et quelques éléments toponymiques dans la ville, cette persistance seigneuriale s'atténue sous le poids des années. Pourtant, plusieurs questions demeurent: y a-t-il un lien direct entre l'abolition du régime seigneurial et la transition de la famille Pozer vers l'administration municipale ? La cohabitation entre seigneurs anglo-protestants et censitaires franco-catholiques a-t-elle été aussi paisible qu'on le prétend ? Cette mémoire favorable à la famille seigneuriale est-elle aussi fréquente dans les autres seigneuries du Québec ? Il est évident que cette introduction à la seigneurie AubertGallion n'a pas la prétention de répondre à toutes ces questions, mais nous pensons tout de même qu'elle pose certains jalons vers des études plus approfondies dirigées par les questions que nous venons d'évoquer. 